\title{
NITRIC OXIDE IMPROVES PULMONARY VASCULAR IMPEDANCE, TRANSPULMONARY EFFICIENCY, AND LEFT VENTRICULAR FILLING IN CHRONIC PULMONARY HYPERTENSION
}

Edward P. Chen, MD

Hartmuth B. Bittner, MD

Frank Tull, BA

Damian Craig, MS

R. Duane Davis, MD

Peter Van Trigt, MD
Objective: Chronic pulmonary hypertension is difficult to treat and despite the introduction of several therapeutic options, no single therapy is universally recommended. Nitric oxide has had some role clinically in improving pulmonary hemodynamics in this setting; however, basic investigation has not been performed in an appropriate large animal model of stable pulmonary hypertension. This study was designed to examine the effects of inhaled nitric oxide on pulmonary hemodynamics in the setting of a canine model of monocrotaline pyrrole-induced chronic pulmonary hypertension and used Fourier analysis for assessment of pulmonary vascular impedance. Methods: Sixteen mongrel dogs ( 22 to $25 \mathrm{~kg}$ ) were used. Animals underwent percutaneous pulmonary artery catheterization to measure right-sided hemodynamics before and 6 weeks after a right atrial injection of either monocrotaline pyrrole $(n=8)$ or placebo $(n=8)$. Six weeks after the injection all hearts were instrumented with an ultrasonic flow probe, sonomicrometric dimension transducers, and micromanometers. Data were collected at baseline and after nitric oxide administration. Harmonic derivation of functional data was achieved with Fourier analysis. Results: Six weeks after the injection, significant increases in pulmonary artery pressure and pulmonary vascular resistance were observed in the monocrotaline pyrrole group. Nitric oxide led to significant decreases in pulmonary vascular impedance. Significant improvements in pulmonary blood flow, transpulmonary efficiency, and left ventricular filling were also observed. Conclusions: This investigation demonstrates the well-known clinical effects of nitric oxide in improving pulmonary hypertension, which were also associated with an increase in pulmonary blood flow, transpulmonary efficiency, and left ventricular filling in the setting of monocrotaline pyrrole-induced pulmonary hypertension. (J Thorac Cardiovasc Surg 1997; 113:849-57)
$T^{\mathrm{h}}$ he clinical management of chronic pulmonary hypertension is challenging and despite the introduction of several therapeutic measures, no single treatment is universally recommended. Numerous

From the Department of Surgery, Division of Cardiovascular and Thoracic Surgery, Duke University Medical Center, Durham, N.C.

Dr. Chen is the recipient of a National Research Service Award, Fellowship No. 1F32HL09489-01.

Received for publication June 24, 1996; revisions requested Oct. 22, 1996; revisions received Nov. 5, 1996; accepted for publication Dec. 27, 1996.

Address for reprints: Edward P. Chen, MD, Box 0470, S-343, Department of Surgery, University of California, San Francisco, San Francisco, CA 94143.

Copyright (C) 1997 by Mosby-Year Book, Inc.

$0022-5223 / 97 \$ 5.00+0 \quad \mathbf{1 2 / 1 / 8 0 1 0 7}$ pharmacologic agents are used as potential strategies for improving pulmonary hemodynamics in this setting, including prostaglandin $\mathrm{E}_{1}$, prostacyclin, and sodium nitroprusside. Unfortunately, these drugs are not selective for the pulmonary vascular bed and, in addition to lowering pulmonary vascular pressure, their use is also associated with significant decreases in the systemic mean arterial pressure. ${ }^{1}$

Nitric oxide (NO) is a selective pulmonary vasodilator that has no significant effects on the systemic circulation. These hemodynamic properties suggest a potentially useful role for NO in improving pulmonary hemodynamics in the setting of chronic pulmonary hypertension. There are few reports, however, that describe the use of NO in reducing pulmonary vascular impedance in this clinical scenario. Furthermore, basic investigation of this prob- 
lem has been limited by lack of an appropriate large-animal model of stable chronic pulmonary hypertension. This study was therefore designed to examine the effects of inhaled NO on pulmonary hemodynamics in the setting of chronic pulmonary hypertension, with use of a recently established canine model of monocrotaline pyrrole-induced chronic pulmonary hypertension ${ }^{2}$ and with Fourier analysis for characterization of pulmonary vascular impedance and blood flow.

\section{Material and methods}

Study design, experimental groups, and drug synthesis. Sixteen adult mongrel dogs weighing 22 to $25 \mathrm{~kg}$ were used and divided into two groups. The experimental group received an injection of $3 \mathrm{mg} / \mathrm{kg}$ of monocrotaline pyrrole (MCTP group, $n=8$ ) whereas control animals received placebo (CTL group, $n=8$ ). Monocrotaline pyrrole was synthesized artificially for use in this study with a welldescribed technique. ${ }^{3}$

Anesthesia, hemodynamic monitoring, and monocrotaline pyrrole injection. All animals were anesthetized with $5 \mathrm{mg} / \mathrm{kg}$ of intravenous thiopental sodium (Gensia Laboratories, Irvine, Calif.) and $20 \mathrm{mg} / \mathrm{kg}$ of intramuscular ketamine sodium (Fort Dodge Laboratories, Fort Dodge, Iowa) before all studies, including baseline and follow-up hemodynamic measurements, drug injections, and NO studies. Each animal also received 900,000 units of penicillin $\mathrm{G}$ benzathine and penicillin $\mathrm{G}$ procaine (Fort Dodge Laboratories). All animals were intubated with a 9F endotracheal tube and the lungs mechanically ventilated with a Bear 1 ventilator (Inter Med, Bear Medical Systems, Inc., Riverside, Calif.). The tidal volume was set at $15 \mathrm{ml} / \mathrm{kg}$, the fraction of inspired oxygen at $21 \%$, and the rate at 10 breaths $/ \mathrm{min}$. Arterial blood samples were drawn from the right femoral artery and processed with a Gem-Stat blood gas analyzer (Mallinckrodt Sensor Systems, Ann Arbor, Mich.).

All hemodynamic measurements and drug injections were done under sterile conditions. For each animal, an $8.5 \mathrm{~F}$ introducer sheath (Baxter Healthcare, Irvine, Calif.) was placed percutaneously into the external jugular vein by the Seldinger technique. A 5F Swan-Ganz catheter (Baxter Healthcare) was then advanced through the introducer sheath into the right atrium, right ventricle, and pulmonary artery for measurement of right atrial, right ventricular, and pulmonary arterial pressures. All pressures were monitored with a Horizon 2000 hemodynamic monitor (Mennen Medical Inc., Clarence, N.Y.). Hemodynamic measurements were done at baseline and 6 weeks after injection. Monocrotaline pyrrole or placebo was injected into the right atrium after measurement of baseline pulmonary hemodynamic indices.

Instrumentation for assessment of cardiopulmonary function. At the time of instrumentation, the fraction of inspired oxygen was increased to $60 \%$ to maintain an arterial partial pressure of oxygen of at least 200 to 300 $\mathrm{mm} \mathrm{Hg}$. A standard median sternotomy and an anterior pericardiotomy were performed to expose the hearts of all animals. An ultrasonic flow probe (T208X, Transonic Systems Inc., Ithaca, N.Y.) was placed around the main pulmonary trunk to measure pulmonary blood flow. Hemispheric ultrasonic dimension transducers $(1.5 \mathrm{~mm}$ outer diameter, No. 1-1015-5A, Vernitron, Bedford, Ohio) were positioned across the base-apex major axis, anteroposterior minor axis diameter of the left ventricle, and septal-free wall minor axis diameters of both the right and left ventricles to measure left and right ventricular cavitary volumes. Micromanometers (MPC-500, Millar Instruments Inc., Houston, Tex.) were placed into the right and left ventricle, right and left atrium, and pulmonary artery for continuous pressure recording of the right and left ventricular pressures, right and left atrial pressures, and pulmonary artery pressure.

NO administration. $\mathrm{NO}$ ( $\mathrm{NO} 777 \mathrm{ppm}$ and $\mathrm{NO}_{2}<0.1$ ppm, National Specialty Gases, Durham, N.C.) was continuously blended into the inspiratory circuit of the ventilator. NO was given in sequential fashion at levels of 40 and $80 \mathrm{ppm}$. The NO and nitric dioxide levels of the inspired gas were measured by continuous chemiluminescent analysis (model 42H, Thermo Environmental Instruments, Inc., Franklin, Mass.).

Experimental protocol during NO studies. Six weeks after monocrotaline pyrrole or placebo injection, baseline postinjection functional and hemodynamic data were collected in every animal in both experimental groups after instrumentation. CTL-group animals did not undergo any further data collection. In the MCTP-group animals, inhaled NO was then administered at levels of 40 and 80 $\mathrm{ppm}$ and data were collected at each concentration. After each incremental increase in the NO concentration, the animal's condition and hemodynamic parameters were allowed to equilibrate for 10 minutes before further data collection. After data were collected at the dose of 80 $\mathrm{ppm}$, the NO administration was discontinued and the level in the inspiratory circuit of the ventilator was restored to $0 \mathrm{ppm}$. Pulmonary hemodynamic parameters were then allowed to return to baseline values.

Data acquisition during baseline and NO studies. All data were digitized on-line, collected, and stored on a microprocessor (PDP 11/23, Digital Equipment Corp., Maynard, Mass.). Pressure data and cardiac output were analyzed with software developed in our laboratory that is described elsewhere. ${ }^{4}$ Briefly, all data were digitized at $500 \mathrm{~Hz}$ and filtered by a $50 \mathrm{~Hz}$ low-pass filter, stored on magnetic media, and analyzed on a Dell Dimension XPS P90 computer (Dell Computer Corp., Austin, Tex.).

Myocardial and lung biopsies. Generous 1.5 to $2 \mathrm{gm}$ peripheral lung tissue samples were taken from the right upper lung lobe of all animals in both the CTL and MCTP groups after median sternotomy and anterior pericardiotomy and analyzed with the use of light microscopy. Hematoxylin and eosin-stained sections were used to assess the pulmonary parenchyma and vasculature in all biopsy samples.

Fourier analysis. Calculation of the impedance spectrum of the pulmonary circulation from the collected raw data was done with Fourier analysis. Fourier analysis is based on the principle that all periodic waveforms may be broken down into a series of pure sine waves or harmonics. $^{5}$ Harmonics exist at frequencies that are multiples of 
the frequency of the original waveform (the "fundamental frequency") and are described in terms of an amplitude and phase. Each harmonic represents an oscillatory component of the original waveform at its respective frequency. The net effect of Fourier analysis is to transform a waveform from the time domain, that is, wherein the waveform is described as a function of time, to the frequency domain, wherein it is described as a function of frequency.

The pulsatile waveforms of the pulmonary artery pressure and flow can be treated as periodic waveforms possessing a fundamental frequency (that is, the heart rate). At each individual harmonic, division of the pressure amplitude by the flow amplitude allows one to obtain the oscillatory counterpart of resistance, impedance, at that respective harmonic. The array of individually calculated impedance values from every harmonic represents the impedance spectrum. Input resistance is the impedance calculated at the zeroth harmonic and is a measure of resistance to mean pulmonary blood flow. Characteristic impedance is estimated as the mean impedance between 2 and $12 \mathrm{~Hz}$ and is a measure of resistance to pulsatile blood flow. In theoretic terms, characteristic impedance is an index of the overall distensibility or stiffness of the pulmonary vascular bed.

The product of the impedance amplitude, the square of the flow amplitude, and the cosine of the impedance phase angle for each individual harmonic give the individual power at that particular harmonic. These products across all harmonics constitute the power spectrum, which represents the amount of energy, or hydraulic power, produced by the right ventricle to maintain pulmonary blood flow and are expressed as follows:

$$
\text { Power }=\sum_{\mathrm{i}} Q^{2} Z \cos \left(\phi_{\mathrm{z}}\right)
$$

where $Q$ represents the flow amplitude, $Z$ represents the impedance amplitude, and $\phi$ represents the impedance phase angle. Hydraulic power can be divided into two components: steady power and oscillatory power. Steady power is the power obtained at the zeroth harmonic and represents the energy required to move blood forward at a steady state. Oscillatory power is taken from the remainder of the power spectrum exclusive of the zeroth harmonic and reflects the energy that is wasted in oscillatory accelerations of blood and that eventually disseminates into vessel walls.

Transpulmonary efficiency was defined as the ratio of mean pulmonary blood flow divided by right ventricular hydraulic power. Pulmonary vascular resistance was calculated by the standard formula applying mean pulmonary artery pressure, left atrial pressure, and cardiac output.

Humane animal care. All animals received humane care in compliance with the "Principles of Laboratory Animal Care" formulated by the National Society for Medical Research and the "Guide for the Care and Use of Laboratory Animals" prepared by the Institute of Laboratory Animal Resources and published by the National Institutes of Health (NIH Publication No. 86-23, revised 1985). The experiments were approved by the Duke
University Institutional Animal Care and Use Committee (DUIACUC Assigned Registry No. A450-94-9).

Statistical analysis. Statistical analysis was performed on a Dell personal computer with commercially available software (SigmaStat, Jandel Corporation, San Rafael, Calif.). Data taken before and after injection of monocrotaline pyrrole within the MCTP group were analyzed with standard two-tailed paired Student's $t$ tests. To test for significant changes in the various parameters of pulmonary vascular impedance after NO administration within the MCTP group, a one-way analysis of variance for repeated measurements was used. Follow-up comparison between hemodynamic parameters at individual levels of NO was done with paired Student's $t$ tests. Unpaired Student's $t$ tests were used to analyze data between the CTL and MCTP groups. The results are expressed as mean plus or minus the standard error of the mean. A difference was considered statistically significant at $p<$ 0.05 .

\section{Results}

There were no significant differences in either the body weights or baseline hemodynamic parameters between the CTL and MCTP groups. In addition, there was no significant change in the body weights of animals in the MCTP group 6 weeks after monocrotaline pyrrole injection compared with before injection.

Hemodynamic changes. Significant increases in the mean right ventricular pressure and mean pulmonary artery pressure were observed in the MCTP group 6 weeks after monocrotaline pyrrole injection $(13.08 \pm 0.57 \mathrm{~mm} \mathrm{Hg}$ and $20.1 \pm 1.10 \mathrm{~mm} \mathrm{Hg}$, respectively, $p<0.001)$ compared with baseline measurements $(8.25 \pm 0.49 \mathrm{~mm} \mathrm{Hg}$ and $9.71 \pm 0.62$ $\mathrm{mm} \mathrm{Hg}$, respectively), whereas no significant changes were observed in heart rate $(96 \pm 4$ beats/ min vs $85 \pm 5$ beats $/ \mathrm{min}$ ) or central venous pressure ( $3.75 \pm 0.45 \mathrm{~mm} \mathrm{Hg}$ vs $3.13 \pm 0.35 \mathrm{~mm} \mathrm{Hg}$ ). In addition, there was a significant increase in the pulmonary vascular resistance in the MCTP group 6 weeks after monocrotaline pyrrole injection compared with values in the CTL group $(951 \pm 129$ dyne $\cdot \mathrm{sec} \cdot \mathrm{cm}^{-5}$ vs $461 \pm 37$ dyne $\cdot \mathrm{sec} \cdot \mathrm{cm}^{-5}, p=$ $0.002)$. A significant increase in the pulmonary capillary wedge pressure was also observed in the MCTP group 6 weeks after monocrotaline pyrrole injection compared with before injection $(6.25 \pm$ $0.75 \mathrm{~mm} \mathrm{Hg}$ vs $4.13 \pm 0.40 \mathrm{~mm} \mathrm{Hg}, p=0.025)$.

Changes in pulmonary vascular mechanics after monocrotaline pyrrole injection. Six weeks after the monocrotaline pyrrole injection, there was a decrease in the pulmonary blood flow from $1384 \pm 87$ $\mathrm{ml} / \mathrm{min}$ in the CTL group to $1236 \pm 92 \mathrm{ml} / \mathrm{min}$ in the MCTP group, although this difference was not sta- 
Table I. Effect of monocrotaline pyrrole-induced chronic pulmonary hypertension on pulmonary vascular mechanics

\begin{tabular}{|c|c|c|c|c|c|c|c|c|c|}
\hline & $\begin{array}{c}Q_{p a} \\
(\mathrm{ml} / \mathrm{min})\end{array}$ & $\begin{array}{c}R I N \\
\left(\text { dyne } \cdot \mathrm{sec} \cdot \mathrm{cm}^{-5}\right)\end{array}$ & $\begin{array}{c}Z_{o} \\
\left(\text { dyne } \cdot \mathrm{sec} \cdot \mathrm{cm}^{-5}\right)\end{array}$ & $\begin{array}{c}H P \\
(m W)\end{array}$ & $\begin{array}{c}S P \\
(m W)\end{array}$ & $\% S P$ & $\begin{array}{c}O P \\
(m W)\end{array}$ & $\% O P$ & $\begin{array}{c}E F F \\
(\mathrm{ml} / \mathrm{min} / \mathrm{mW})\end{array}$ \\
\hline $\operatorname{CTL}(n=8)$ & $1384(87)$ & $685(59)$ & $223(21)$ & $50(3.0)$ & $35(2.5)$ & $71(2.7)$ & $15(1.5)$ & $29(2.7)$ & $36.2(2.3)$ \\
\hline $\operatorname{MCTP}(n=8)$ & $1236(92)$ & $1325(137)^{*}$ & $227(32)$ & $68(7.6) \dagger$ & $54(5.3) \div$ & $80(1.9) \S$ & $14(2.6)$ & $20(1.9) \S$ & $24.9(2.5) \|$ \\
\hline
\end{tabular}

The standard error of the mean is given in parentheses. $Q_{p w}$ Pulmonary blood flow; $R I N$, input resistance; $Z_{o}$, characteristic impedance; $H P$, hydraulic power; $S P$, steady power; $\% S P$, percentage of hydraulic power comprising steady power; $O P$, oscillatory power; $\% O P$, percentage of hydraulic power comprising oscillatory power; $E F F$, transpulmonary efficiency.

${ }^{*} p<0.001$ versus CTL.

$t_{p}=0.035$ versus CTL.

$\uparrow p=0.005$ versus CTL.

$\S p=0.013$ versus CTL.

$\| p=0.001$ versus CTL.

tistically significant. There were significant increases in both input resistance and right ventricular hydraulic power in the MCTP group compared with values in the CTL group. Steady power was also significantly increased, whereas transpulmonary efficiency was significantly decreased in the MCTP group compared with that in the CTL group. No significant differences were observed in the characteristic impedance or oscillatory power between the two groups 6 weeks after the injection. The changes in pulmonary vascular mechanics after monocrotaline pyrrole injection are summarized in Table I.

Effect of inhaled NO on pulmonary vascular mechanics. After NO administration in the MCTP group, the mean pulmonary artery pressure decreased significantly at both $40 \mathrm{ppm}$ and $80 \mathrm{ppm}$ compared with the baseline value, although no further significant decrease was observed in the mean pulmonary artery pressure at $80 \mathrm{ppm}$ of NO compared with that at $40 \mathrm{ppm}$ (Fig. 1). The pulmonary vascular resistance was also significantly decreased after NO administration compared with the baseline value (Fig. 2). These levels after NO administration were not significantly different compared with preinjection levels.

NO led to significant increases in both mean pulmonary blood flow and transpulmonary efficiency in the MCTP group at $40 \mathrm{ppm}$ and $80 \mathrm{ppm}$ compared with values at $0 \mathrm{ppm}$. Left atrial pressure and left ventricular filling were also significantly increased at 80 $\mathrm{ppm}$ NO compared with values at $0 \mathrm{ppm}$. In addition, there were significant decreases in the input resistance at $40 \mathrm{ppm}$ and $80 \mathrm{ppm}$ compared with the value at 0 $\mathrm{ppm}$. No significant differences in the right ventricular hydraulic power, steady power, or oscillatory power were observed after NO administration. The effects of inhaled NO on pulmonary vascular mechanics are summarized in Table II.
Gross and microscopic lung tissue analysis. Gross examination of the lungs in the MCTP group revealed no obvious abnormalities compared with CTL-group lungs. Microscopic examination of hematoxylin and eosin-stained lung sections in the CTL group revealed normal thin-walled pulmonary arterioles and alveolar septae. Lung sections from all experimental animals in the MCTP group demonstrated thickening of the small and medium pulmonary arterioles with smooth muscle hypertrophy within the vessel walls in every specimen (Fig. 3). There was also increased cellularity and widening of the alveolar septae. The majority of the small pulmonary arterioles observed were nearly obliterated because of increased amounts of stromal tissue and fibrous connective tissue surrounding these vessels.

\section{Discussion}

NO is an endogenously occurring endotheliumderived relaxing factor produced from $\mathrm{L}$-arginine by endothelial NO synthetase. ${ }^{6,7}$ Whether produced by the endothelial cells or after exogenous administration by inhalation, NO rapidly diffuses directly into the pulmonary vascular smooth muscle cells where it leads to increases in the concentration of guanylate cyclase. ${ }^{8}$ The rise in the level of guanylate cyclase results in relaxation and vasodilation of the pulmonary vascular smooth muscle. Once NO diffuses through the smooth muscle cell into the vessel lumen, however, it reacts quickly with hemoglobin. Hemoglobin inactivates NO and prevents it from causing any systemic vasodilation. In this way, the vasodilating actions of inhaled NO are focused on the pulmonary circulation with virtually no systemic effects. ${ }^{9}$ These properties offer a significant advantage for NO as a selective pulmonary vasodilator.

Clinically, NO has successfully improved pulmonary hemodynamics in primary pulmonary hyper- 


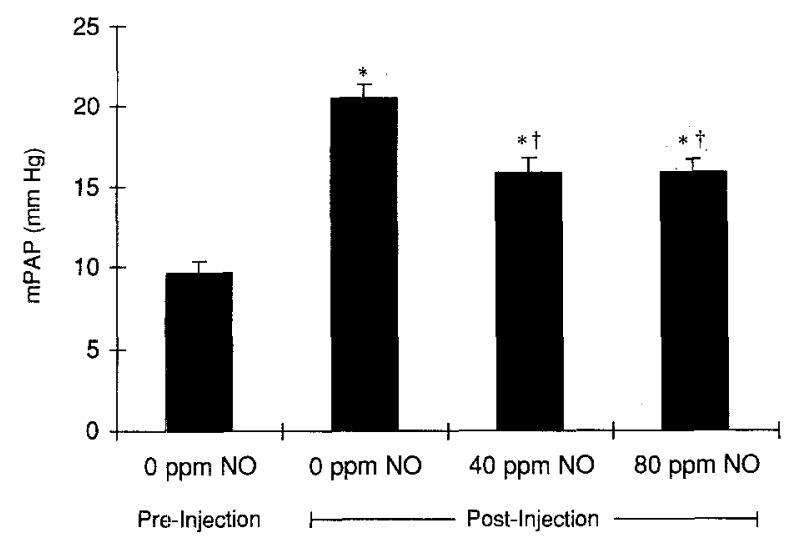

Fig. 1. Bar graph displaying the significant increase in the mean pulmonary artery pressure $(m P A P)$ in the monocrotaline pyrrole-treated group 6 weeks after injection compared with before injection and the effects of NO administration. Six weeks after monocrotaline pyrrole injection, significant decreases in the mean pulmonary artery pressure were observed after NO administration, although these posttreatment levels continued to be significantly elevated compared with those before injection. * $p<0.001$ versus preinjection; ${ }^{\dagger} p<0.001$ versus postinjection, $0 \mathrm{ppm}$ NO.

tension $^{10}$ and in pulmonary hypertension caused by congenital heart defects, ${ }^{11}$ mitral valve disease, ${ }^{12}$ and adult respiratory distress syndrome, ${ }^{13}$ as well as after cardiac operations. ${ }^{14} \mathrm{NO}$ has also been used in the treatment of chronic pulmonary hypertension in patients with congestive heart failure ${ }^{15}$ and in the evaluation and identification of reversible pulmonary hypertension in patients awaiting cardiac transplantation. ${ }^{16}$

In the experimental setting, NO has been reported to lower pulmonary artery pressure, albeit in the setting of acute pulmonary hypertension, in animal models of acute hypoxia, ${ }^{17}$ endotoxin shock, ${ }^{18}$ and acute lung injury. ${ }^{19}$ Few reports, however, describe the experimental use of $\mathrm{NO}$ for improving pulmonary hemodynamics in the setting of chronic pulmonary hypertension.

This report brings several novel approaches to basic investigation of the effects of NO on pulmonary hemodynamics in the setting of chronic pulmonary hypertension. First, a recently established canine model of monocrotaline pyrrole-induced chronic pulmonary hypertension was used. Several other models of pulmonary hypertension exist as potential means to evaluate the different pharmacologic options for improving pulmonary hemodynam-

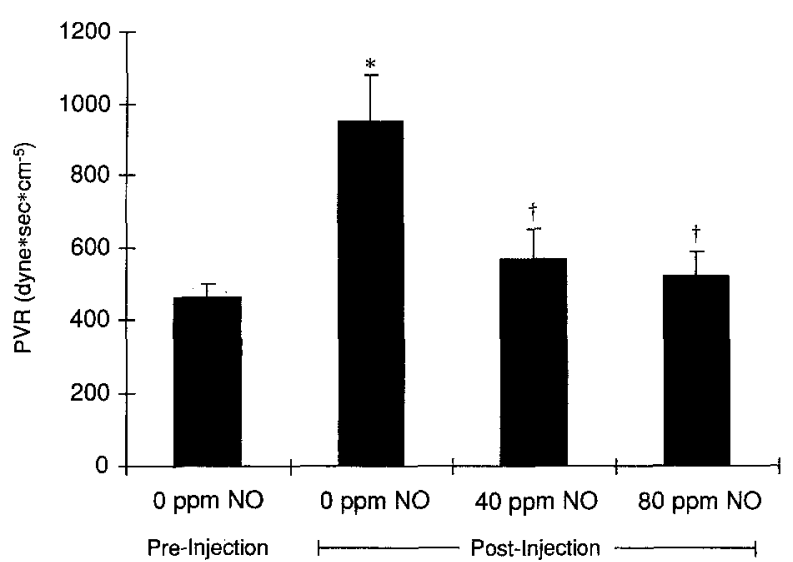

Fig. 2. Bar graph displaying the significant increase in the pulmonary vascular resistance $(P V R)$ in the monocrotaline pyrrole-treated group 6 weeks after injection compared with before injection and the effects of NO administration. Six weeks after injection of monocrotaline pyrrole, significant decreases in the pulmonary vascular resistance were observed after NO administration to levels that were not significantly different from preinjection values. ${ }^{*} p=0.002$ versus preinjection; ${ }^{\dagger} p<0.001$ versus postinjection, 0 ppm NO.

ics in the setting of chronically elevated pulmonary vascular pressure. These models use a variety of methods to induce pulmonary hypertension, including embolization, ${ }^{20}$ banding of the pulmonary artery, $^{21}$ and pulmonary venous constriction. ${ }^{22} \mathrm{Al}-$ though each previous model resulted in a significant rise in pulmonary vascular pressure, the pressure increases were accomplished in an acute manner and do not mimic the chronic nature of chronic pulmonary hypertension. In addition, the parenchymal changes seen in association with primary pulmonary hypertension were not reported in any previous model. As a result, these models do not adequately represent the clinical picture of chronic pulmonary hypertension.

Monocrotaline pyrrole-induced chronic pulmonary hypertension is characterized by a proliferative vasculitis. ${ }^{23}$ After intravenous injection, the parent compound, monocrotaline, undergoes hepatic metabolism by the cytochrome P450 monooxygenase system to form a reactive metabolite, monocrotaline pyrrole, which in turn circulates to the pulmonary vascular bed where lung injury is induced. The initial endothelial injury leads to increased capillary permeability, which results in interstitial and alveolar edema. $^{23}$ Remodeling of the pulmonary vascular 


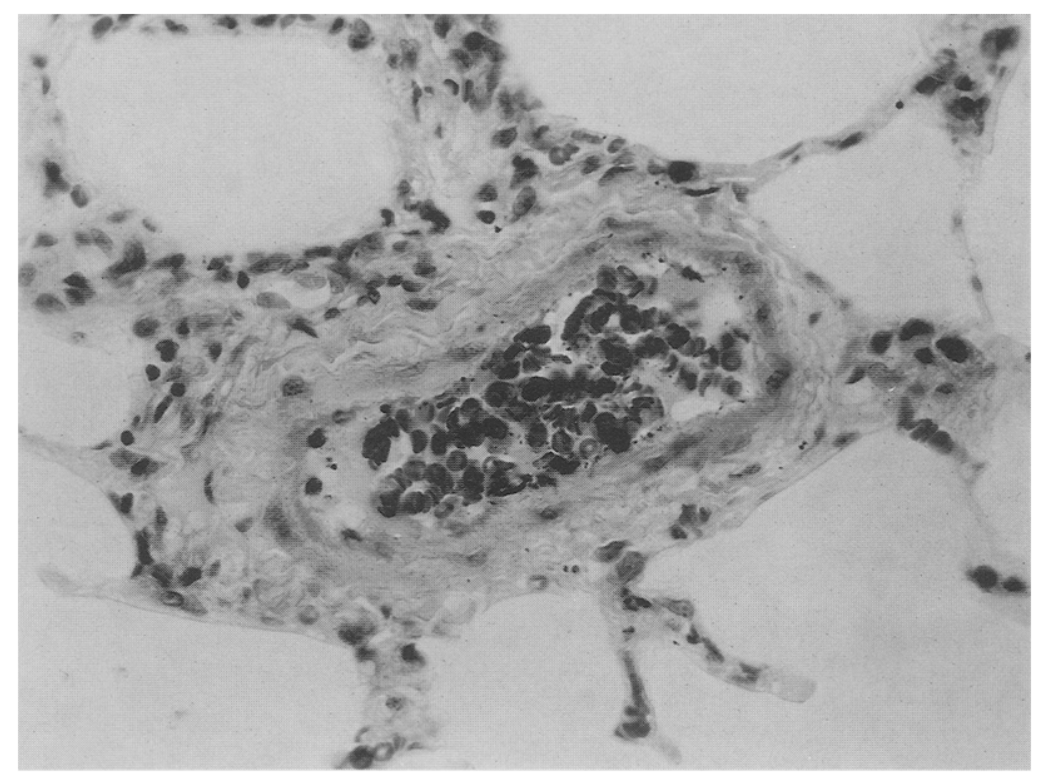

Fig. 3. Photomicrograph of a hematoxylin and eosin-stained specimen of lung tissue taken from an experimental animal 6 weeks after monocrotaline pyrrole injection illustrating plexiform changes in the arterioles. There is increased connective tissue, smooth muscle proliferation, and adventitia surrounding the medium-sized pulmonary arteriole.

Table II. Effect of inhaled NO on pulmonary vascular mechanics in setting of monocrotaline pyrrole-induced chronic pulmonary hypertension

\begin{tabular}{|c|c|c|c|c|c|c|c|c|c|c|c|c|}
\hline & $\begin{array}{c}N O \\
(\mathrm{ppm}) \\
\end{array}$ & $\begin{array}{c}Q_{p a} \\
(\mathrm{ml} / \mathrm{min})\end{array}$ & $\begin{array}{c}\text { RIN } \\
(\text { dyne } \cdot \\
\left.\mathrm{sec} \cdot \mathrm{cm}^{-5}\right)\end{array}$ & $\begin{array}{c}Z_{o} \\
(\text { dyne } \cdot \\
\left.\sec \cdot \mathrm{cm}^{-5}\right)\end{array}$ & $\begin{array}{c}H P \\
(m W)\end{array}$ & $\begin{array}{c}S P \\
(m W)\end{array}$ & $\% S P$ & $\begin{array}{c}O P \\
(m W)\end{array}$ & $\% O P$ & $\begin{array}{c}E F F \\
(\mathrm{ml} / \mathrm{min} / \mathrm{mW})\end{array}$ & $\begin{array}{c}L A P \\
(m m H g)\end{array}$ & $\begin{array}{l}L V V \\
(m l)\end{array}$ \\
\hline $\begin{array}{l}\text { MCTP } \\
\quad(n=8)\end{array}$ & 0 & $1236(92)$ & $1325(137)$ & $227(32)$ & $68(7.6)$ & $54(5.3)$ & $80(1.9)$ & $14(2.6)$ & $20(1.9)$ & $24.9(2.5)$ & $5.7(0.6)$ & $46(4.4)$ \\
\hline $\begin{array}{l}\text { MCTP } \\
\quad(n=8)\end{array}$ & 40 & $1455(140)^{*}$ & $976(128) \div$ & $260(23)$ & $70(9.6)$ & $55(7.6)$ & $79(1.0)$ & $15(2.1)$ & $21(1.0)$ & $33.4(3.5) \ddagger$ & $7.02(0.4)$ & $49(5)$ \\
\hline $\begin{array}{l}\text { MCTP } \\
(n=8)\end{array}$ & 80 & $1469(115)^{*}$ & $945(85) \dagger$ & $250(39)$ & $72(8.8)$ & $56(7.1)$ & $77(1.6)$ & $16(2.0)$ & $23(1.6)$ & $33.2(3.2) \neq$ & $7.55(0.4) \S$ & $51(4.9) \|$ \\
\hline
\end{tabular}

The standard exror of the mean is given in parentheses. $Q_{p o}$ Pulmonary blood flow; $R I N$, input resistance; $Z_{o}$, characteristic impedance; $H P$, hydraulic power; $S P$, steady power; $\% S P$, percentage of hydraulic power comprising steady power; $O P$, oscillatory power; $\% O P$, percentage of hydraulic power comprising oscillatory power; $E F F$, transpulmonary efficiency; $L A P$, left atrial pressure; $L V V$, left ventricular volume.

${ }^{*} p=0.021$ versus MCTP, 0 ppm NO.

$\ddagger p=0.003$ versus MCTP, $0 \mathrm{ppm}$ NO.

${ }^{p}<0.001$ versus MCTP, 0 ppm NO.

$\$ p=0.014$ versus MCTP, $0 \mathrm{ppm}$ NO.

$\| p=0.030$ versus MCTP, 0 ppm NO.

bed at the level of the medium and small pulmonary arterioles, which occurs in response to this injury, is responsible for the rise in hemodynamic pressures.

Light microscopy examination of lung biopsy specimens from animals treated with monocrotaline pyrrole revealed endothelial cell degeneration and hyperplasia and medial smooth muscle hypertrophy with subsequent medial thickening affecting both the small and medium arterioles. These findings closely mimic the changes observed in a pathologic type of primary pulmonary hypertension known as plexogenic pulmonary arteriopathy. ${ }^{24}$ Thus the model of pulmonary hypertension described in this report accurately reflects the clinical picture of primary pulmonary hypertension not only because it is chronic, but also, more important, because the parenchymal changes that occur as a result of monocrotaline pyrrole injection are similar to the microscopic findings observed clinically in chronic pulmonary hypertension. 
A possible criticism of this model is that the absolute level of pulmonary hypertension observed after monocrotaline pyrrole injection is not reflective of the clinical picture of chronic pulmonary hypertension. It is important to note, however, that the pulmonary vascular pressures observed in the canines before drug injection in these experiments were significantly lower than normal levels found in a human being. Although a twofold increase in the mean pulmonary artery pressure and pulmonary vascular resistance was achieved, the resultant levels are, admittedly, still lower than those usually seen clinically. It is unclear from the data presented in this report whether any further significant increase in pulmonary hemodynamic values would occur at more than 6 weeks after monocrotaline pyrrole injection, and this will require further study. A potential way to refine this model and possibly achieve a higher degree of pulmonary hypertension would be to perform numerous injections of monocrotaline pyrrole at regularly spaced intervals. Multiple injections in the same animal would theoretically cause repeated injury to the pulmonary vascular bed and produce greater scarring of the pulmonary vasculature, resulting in higher pulmonary pressures.

Admittedly, this model does not precisely reflect the exact clinical picture of chronic pulmonary hypertension, although the picture it does provide succeeds where previous models have failed. Clearly, no single experimental model could possibly replicate the entire pattern of chronic pulmonary hypertension. Therefore the choice of any one established or new animal model should be based on the need to answer a particular question without attempting to produce an exact replica of any one single disease process.

This report is also unique in that Fourier analysis was used to calculate pulmonary vascular impedance and right ventricular hydraulic power. The pulmonary vascular bed represents a highly compliant, low-resistance conduit that is subject to pulsatile blood flow. Traditional measurements of steadystate hemodynamic parameters, such as mean pulmonary artery pressure, pulmonary vascular resistance, and mean pulmonary artery blood flow, do not fully characterize the nature of the pulmonary circulation in terms of its physical state. The application of Fourier analysis to the parameters of pulmonary artery pressure and blood flow in this report separates these measurements into their respective steady-state and pulsatile components, re- sults in a complete and quantitative description of the pulmonary vascular bed in the form of vascular impedance, gives information regarding the physical state of the pulmonary vasculature, and thoroughly assesses the effects of NO on these components. The interactions of the right ventricle and its afterload can also be assessed with Fourier analysis to precisely define the external load against which the right ventricle must pump to move blood forward through the pulmonary circulation. This analysis allows for determination of right ventricular hydraulic power and divides this power into its components of steady power and oscillatory power. The use of Fourier analysis to assess biologic systems has been previously investigated and validated for the pulmonary vascular systems in both canine and human models. ${ }^{25}$

The smooth muscle hypertrophy and perivascular connective tissue proliferation observed in the small and medium arterioles of all experimental animals treated with monocrotaline pyrrole may account for the significant increases in pulmonary vascular impedance and right ventricular hydraulic power and the significant decreases in transpulmonary efficiency. These findings suggest that vascular injury caused by monocrotaline pyrrole leads to a situation in which the pulmonary circulation does not allow blood to flow as easily or efficiently. On several occasions, the increased vascular cellular hyperplasia observed in the pulmonary specimens was so pronounced that the vessel lumen was nearly, if not completely, occluded. These microscopic changes led to decreased vascular compliance and increased right ventricular afterload. Significantly greater amounts of energy expenditure were required by the right ventricle to maintain the same degree of pulmonary blood flow. This forward blood flow was maintained at a steady state, but was achieved at a significantly lower level of efficiency.

In addition to the significant improvements in conventional steady-state flow hemodynamics, NO led to only slight increases in right ventricular hydraulic power and insignificant changes in both the steady power and oscillatory power. Thus, in this model of pulmonary hypertension, the beneficial effects of NO appear to be related to its wellestablished ability to selectively dilate the pulmonary circulation. By exclusively reducing pulmonary impedance and hence the external load against which the right ventricle must pump, NO resulted in significant increases in pulmonary blood flow and transpulmonary efficiency, all without acting directly 
on the myocardium as a positive inotrope or increasing the energy expenditure of the right ventricle. These improvements in pulmonary blood flow characteristics, in turn, led to the significant increases in left ventricular filling pressures.

The significant increases that occurred in left atrial pressure and left ventricular volume after NO administration were interesting and surprising findings in this report. These observations are, however, consistent with those in previous investigations in which an increase in the pulmonary capillary wedge pressure was associated with a decrease in pulmonary vascular resistance during infusion of adenosine in patients with heart failure. ${ }^{26}$ Although the exact mechanism for this observation remains unclear, it appears that left ventricular preload increased as a result of the significantly greater amount of blood passing through the pulmonary circulation to the left side of the heart.

The improvements in pulmonary vascular impedance, transpulmonary efficiency, and left ventricular filling after NO administration suggest that the pulmonary circulation is responsive to pharmacologic therapy after monocrotaline pyrrole-induced pulmonary vascular injury and that irreversible damage has not occurred. Interestingly, in a previous study examining the role of NO in the setting of monocrotaline-induced chronic pulmonary hypertension, Mathew and associates ${ }^{27}$ demonstrated that by 2 weeks after monocrotaline injection NO-related relaxation was significantly impaired compared with baseline data in a rat model. In that investigation, exposure of pulmonary artery rings to both L-arginine and acetylcholine failed to result in any vasorelaxation.

The rat model of monocrotaline-induced pulmonary hypertension has been extensively described in the literature. The main disadvantage to the use of rats is the small body size, which makes it technically difficult to accurately assess pulmonary hemodynamics and ventricular volumes in a chronic pulmonary hypertension model. More important, however, the pulmonary circulation of a rat is quite different from that of a human being with respect to vessel ultrastructure, remodeling patterns, and pathologic reactions to injury, which makes it a poor animal model for the study of human chronic pulmonary hypertension. ${ }^{28}$

The findings of Mathew and associates ${ }^{27}$ suggest that irreversible pulmonary vascular injury occurred after monocrotaline treatment and that no significant hemodynamic response to NO should be ex- pected, which apparently contradicts the findings reported in this study. It is important to note, however, that NO-mediated vasorelaxation can occur through both endothelium-dependent, via endogenous production, and endothelium-independent mechanisms. In the study by Mathew and associates, ${ }^{27}$ vasorelaxation of pulmonary vascular rings was attempted with acetylcholine. Acetylcholine, in addition to L-arginine as previously mentioned, mediates vasodilation by an endotheliumdependent pathway, ${ }^{29}$ and failure to observe any pulmonary vasorelaxation in this particular circumstance would certainly be consistent with the documented mechanisms of monocrotaline pyrrole-induced pulmonary endothelial injury.

In the present study, NO was directly administered into the alveolar air spaces. When given in this manner, NO diffuses across the alveolar-capillary membrane to increase guanylate cyclase levels in vascular smooth muscle, causing pulmonary vasodilation, and directly bypasses the endothelial cells. Thus the improvement in pulmonary vascular impedance observed in this report occurred through an endothelium-independent mechanism, and in the setting of monocrotaline pyrrole-induced pulmonary endothelial injury it appears that mechanisms of pulmonary vasodilation via vascular smooth muscle cells are at least partially intact.

Because of the previously well-described injury to pulmonary vascular endothelial cells and the subsequent changes that occur in the vascular smooth muscle as a result of monocrotaline pyrrole injection, it was initially unclear whether the endothelium-dependent and the endothelium-independent mechanisms of NO-mediated vasorelaxation would be altered. Consequently, it was decided to use a relatively high concentration of NO in an attempt to potentially demonstrate a significant hemodynamic response. Although lower concentrations of NO, such as $10 \mathrm{ppm}$ or $20 \mathrm{ppm}$, may be equally effective in reducing vascular impedance as the concentrations used in this report, such a conclusion is not possible on the basis of the data presented in this study. Future studies should examine whether 10 ppm or $20 \mathrm{ppm}$ NO would be effective in improving pulmonary vascular impedance and efficiency in the setting of canine chronic pulmonary hypertension caused by monocrotaline pyrrole.

It is doubtful that any improvements in pulmonary hemodynamics in this model could be achieved by simply increasing the fraction of inspired oxygen while simply ventilating the lungs of animals previ- 
ously given an injection of monocrotaline pyrrole, because the fraction of inspired oxygen was kept at least at $60 \%$ at the time of instrumentation. This was done to maximally stabilize the condition of the animals during the extremely invasive surgical procedures required for placement of all devices used for data collection in these studies.

In summary, an adult canine model of chronic pulmonary hypertension provides a useful and reliable means by which to evaluate the effects of inhaled NO on pulmonary hemodynamics in the setting of chronically elevated pulmonary vascular pressure. The wellknown clinical effects of $\mathrm{NO}$ in improving pulmonary hemodynamics were observed and, in the setting of monocrotaline pyrrole-induced chronic pulmonary hypertension, were also associated with significant improvernents in transpulmonary efficiency and left ventricular filling. These data suggest that NO may be a potentially useful means for significantly improving pulmonary vascular impedance and efficiency in the setting of chronic pulmonary hypertension.

\section{REFERENCES}

1. Kieler-Jensen N, Lundin S, Ricksten SE. Vasodilator therapy after heart transplantation: effects of inhaled nitric oxide and intravenous prostacyclin, prostaglandin $\mathrm{E}_{1}$, and sodium nitroprusside. J Heart Lung Transplant 1995;14:436-43.

2. Chen EP, Bittner HB, Tull F, Biswas S, Davis RD, Van Trigt $P$. An adult canine model of chronic pulmonary hypertension for cardiopulmonary transplantation. J Heart Lung Transplant. In press.

3. Mattocks AR, Jukes R, Brown J. Simple procedures for preparing putative toxic metabolites of pyrrolizidine alkaloids. Toxicon 1989;27:561-7.

4. Glower DD, Spratt JA, Snow ND, et al. Linearity of the Frank-Starling relationship in the intact heart: the concept of preload recruitable stroke work. Circulation 1985;71:9941009.

5. Milnor WR. Hemodynamics. 2nd ed. Baltimore: Williams and Wilkins, 1989:167-203.

6. Ignarro LJ, Buga GM, Wood KS, Byrns RE, Chaudhuri G. Endothelium-derived relaxing factor produced and released from artery and vein is nitric oxide. Proc Natl Acad Sci 1987;84:9265-9.

7. Palmer RMJ, Ferrige AG, Moncada S. Nitric oxide release accounts for the biological activity of endothelium-derived relaxing factor. Nature 1987;327:524-6.

8. Ignarro L. Biological actions and properties of endothelialderived nitric oxide formed and released from artery and vein. Circ Res 1989;65:1-21.

9. Lunn RJ. Inhaled nitric oxide therapy. Mayo Clin Proc $1995 ; 70: 247-55$.

10. Pepke-Zaba J, Higenbottam TW, Dinh-Xuan AT, Stone D, Walkwork J. Inhaled nitric oxide as a cause of selective pulmonary vasodilation in pulmonary hypertension. Lancet 1991;338:1173-4.

11. Roberts JD, Lang P, Bigatello LM, Vlahakes GJ, Zapol WM.
Inhaled nitric oxide in congenital heart disease. Circulation 1993;87:447-53.

12. Girard C, Lehot JJ, Pannetier JC, Filley S, French P, Estanove $S$. Inhaled nitric oxide after mitral valve replacement in patients with chronic pulmonary artery hypertension. Anesthesiology 1992;77:880-3.

13. Rossaint R, Falke KJ, Lopez F, Slama K, Pison U, Zapol WM. Inhaled nitric oxide for the adult respiratory distress syndrome. N Engl J Med 1993;328:399-405.

14. Fullerton DA, Jones SD, Jaggers J, Piedalue F, Grover FL, McIntyre RC. Effective control of pulmonary vascular resistance with inhaled nitric oxide after cardiac operation. J Thorac Cardiovasc Surg 1996;111:753-63.

15. Semigran MJ, Cockrill BA, Kacmarek R, et al. Hemodynamic effects of inhaled nitric oxide in heart failure. J Am Coll Cardiol 1994;24:982-8.

16. Adatia I, Perry S, Landzberg M, Moore P, Thompson JE, Wessel DL. Inhaled nitric oxide and hemodynamic evaluation of patients with pulmonary hypertension before transplantation. J Am Coll Cardiol 1995;25:1656-64.

17. Frostell C, Fratacci MD, Wain JC, Jones R, Zapol WM. Inhaled nitric oxide: a selective pulmonary vasodilator reversing hypoxic pulmonary vasoconstriction. Circulation 1991;83: 2038-47

18. Weitzberg E, Rudehill A, Lundberg JM. Nitric oxide inhalation attenuates pulmonary hypertension and improves gas exchange in endotoxin shock. Eur J Pharmacol 1993;233:8594.

19. Hillman ND, Meliones JN, Black DR, Craig DM, Cheifetz IM, Smith PK. In acute lung injury, inhaled nitric oxide improves ventilation-perfusion matching, pulmonary vascular mechanics, and transpulmonary vascular efficiency. J Thorac Cardiovase Surg 1995;110:593-600.

20. McLean RF, Prielipp RC, Rosenthal MH, Pearl DG. Vasodilator therapy in microembolic porcine pulmonary hypertension. Anesth Analg 1990;71:35-41.

21. Hsieh CM, Mishkel GJ, Cardoso PFG, et al. Production and reversibility of right ventricular hypertrophy and right heart failure in dogs. Ann Thorac Surg 1992;54:104-10.

22. Silore ED, Tavernor WD, Berry CL. Reactive pulmonary arterial hypertension after pulmonary venous constriction in the calf. Cardiovasc Res 1972;6:36-44.

23. Roth RA, Reindel JF. Lung vascular injury from monocrotaline pyrrole, a putative hepatic metabolite. Adv Exp Med Biol 1991;283:477-87.

24. Edwards W, Edwards J. Clinical primary pulmonary hypertension: three pathologic types. Circulation 1977;56:884-8.

25. Attinger EO, Anne A, McDonald DA. Use of Fourier series for the analysis of biological systems. Biophys J 1966;6:291304.

26. Haywood G, Sneddon J, Bashir Y, Jennison S, Gray H, McKenna W. Adenosine infusion for the reversal of pulmonary vasoconstriction in biventricular failure, a good test but a poor therapy. Circulation 1992;86:969-92.

27. Mathew R, Zeballos GA, Tun H, Gewitz MH. Role of nitric oxide and endothelin-1 in monocrotaline-induced pulmonary hypertension in rats. Cardiovasc Res 1995;30:739-46.

28. Heath D. The rat is a poor animal model for the study of human pulmonary hypertension. Cardioscience 1992;3:1-6.

29. Furchgott RF, Zawadzki JV. The obligatory role of endothelial cells in the relaxation of arterial smooth muscle by acetylcholine. Nature 1980;288:373-6. 